\title{
Testing Cosmological Models with Type Ic Super Luminous Supernovae
}

\author{
Jun-Jie Wei ${ }^{1,3}$, Xue-Feng $\mathrm{Wu}^{1,4,5}$ and Fulvio Melia ${ }^{1,2}$
}

Received

accepted

${ }^{1}$ Purple Mountain Observatory, Chinese Academy of Sciences, Nanjing 210008, China.

${ }^{2}$ Department of Physics, The Applied Mathematics Program, and Department of Astronomy, The University of Arizona, AZ 85721, USA; fmelia@email.arizona.edu.

${ }^{3}$ University of Chinese Academy of Sciences, Beijing 100049, China; jjwei@pmo.ac.cn.

${ }^{4}$ Chinese Center for Antarctic Astronomy, Nanjing 210008, China; xfwu@pmo.ac.cn.

${ }^{5}$ Joint Center for Particle, Nuclear Physics and Cosmology, Nanjing University-Purple Mountain Observatory, Nanjing 210008, China. 


\begin{abstract}
The use of type Ic Super Luminous Supernovae (SLSN Ic) to examine the cosmological expansion introduces a new standard ruler with which to test theoretical models. The sample suitable for this kind of work now includes 11 SLSN Ic's, which have thus far been used solely in tests involving $\Lambda$ CDM. In this paper, we broaden the base of support for this new, important cosmic probe by using these observations to carry out a one-on-one comparison between the $R_{\mathrm{h}}=c t$ and $\Lambda \mathrm{CDM}$ cosmologies. We individually optimize the parameters in each cosmological model by minimizing the $\chi^{2}$ statistic. We also carry out Monte Carlo simulations based on these current SLSN Ic measurements to estimate how large the sample would have to be in order to rule out either model at a $\sim 99.7 \%$ confidence level. The currently available sample indicates a likelihood of $\sim 70-80 \%$ that the $R_{\mathrm{h}}=c t$ Universe is the correct cosmology versus $\sim 20-30 \%$ for the standard model. These results are suggestive, though not yet compelling, given the current limited number of SLSN Ic's. We find that if the real cosmology is $\Lambda$ CDM, a sample of $\sim 240$ SLSN Ic's would be sufficient to rule out $R_{\mathrm{h}}=c t$ at this level of confidence, while $\sim 480$ SLSN Ic's would be required to rule out $\Lambda \mathrm{CDM}$ if the real Universe is instead $R_{\mathrm{h}}=c t$. This difference in required sample size reflects the greater number of free parameters available to fit the data with $\Lambda$ CDM. If such SLSN Ic's are commonly detected in the future, they could be a powerful tool for constraining the dark-energy equation of state in $\Lambda \mathrm{CDM}$, and differentiating between this model and the $R_{\mathrm{h}}=c t$ Universe.
\end{abstract}

Subject headings: cosmic background radiation - cosmological parameters - cosmology: observations - cosmology: theory - distance scale - supernovae: general 


\section{Introduction}

A new type of luminous transient has been identified in recent years through the use of deep, wide surveys searching for supernovae in the local Universe (Quimby et al. 2005, 2007; Quimby 2006; Smith et al. 2007; Drake et al. 2009; Rau et al. 2009; Kaiser et al. 2010; Gal-Yam 2012; Baltay et al. 2013). These super-luminous supernovae (SLSN) have peak magnitudes $M_{A B}<-21 \mathrm{mag}$ and integrated burst energies $\sim 10^{51} \mathrm{erg}$. They are therefore much brighter than both Type Ia SNe and the majority of core-collapse events. Their unusually high peak luminosities, hot blackbody temperatures and bright rest frame ultra-violet emission (which renders their continuum easily detectable at optical and near-infrared wavelengths at high redshifts; Cooke et al. 2012) make them viable standardizable candles and distance indicators, and several propoals have already been made to use them as cosmological probes (Li et al. 2014; Cano \& Jakobsson 2014; Inserra \& Smartt 2014).

Inserra et al. (2013) and Nicholl et al. (2013) used the classification term SLSN Ic to refer to all the hydrogen poor SNLSe, though there appear to be at least two observational groups in this category. These are distinguished via the terms SN2005ap-like and SN2007bi-like events, since these are the prototypes with the faster and slower evolving lightcurves. SLSN Ic's have now been discovered out to redshifts $z \sim 4$ (Chomiuk et al .2011; Cooke et al. 2012; Berger et al. 2012), and appear to be rather homogeneous in their spectroscopic and photometric properties.

The brighter events decline more slowly, not unlike the Phillips relation for Type Ia SNe, which raises the possibility of correlating their peak magnitudes to their decline over a fixed number of days to reduce the scatter (Rust 1974; Pskovskii 1977; Phillips 1993; Hamuy et al. 1996). Without the use of such a relation, the uncorrected raw mean magnitudes show a dispersion of $\sim 0.4$ (e.g., Inserra \& Smartt 2014). Correlating the peak 
magnitude to the decline over 30 days reduces the scatter in standardized peak magnitudes to \pm 0.22 mag. And apparently using a magnitude-color evolution reduces this scatter even more, to a low value somewhere between \pm 0.08 mag and \pm 0.13 mag.

It is therefore quite evident that SLSN Ic's may be useful cosmological probes, perhaps even out to redshifts much greater $(z>>2)$ than those accessible using Type Ia SNe. The currently available sample, however, is still quite small; adequate data to extract correlations between empirical, observable quantities, such as lightcurve shape, color evolution and peak luminosity, are available only for tens of events. Our focus in this paper is specifically to study whether SLSN Ic's can be used - not only to optimize the parameters in $\Lambda$ CDM (Li et al. 2014; Cano \& Jakobsson 2014; Inserra \& Smartt 2014), e.g., to refine the dark-energy equation of state but, also - to carry out comparative studies between competing cosmologies, such as $\Lambda \mathrm{CDM}$ versus the $R_{\mathrm{h}}=c t$ Universe (Melia 2007; Melia \& Abdelqader 2009; Melia \& Shevchuk 2012; Melia 2013a).

The application of model selection tools in one-on-one comparisons between these two cosmologies has shown that the data tend to favor $R_{\mathrm{h}}=c t$ over $\Lambda$ CDM. Tests completed thus far include high- $z$ quasars (Melia 2013b, 2014), gamma ray bursts (Wei et al. 2013), the use of cosmic chronometers (Melia \& Maier 2013) and, most recently, the Type Ia SNe themselves (Wei et al. 2014). The $R_{\mathrm{h}}=c t$ and $\Lambda$ CDM cosmologies are very similar, except that the former adopts the additional constraint $p=-\rho / 3$ on its total equation of state, in terms of the total pressure $p$ and energy density $\rho$. In these tests, information criteria show that $R_{\mathrm{h}}=c t$, with this important additional constraint on its equation of state, is favored over $\Lambda$ CDM with a likelihood of $\sim 90 \%$ versus only $\sim 10 \%$.

Here, we broaden the comparison between $R_{\mathrm{h}}=c t$ and $\Lambda$ CDM by now including SLSN Ic's in this study. In $\S 2$ we briefly describe the currently available sample and our method of analysis. Our results are presented in $\S 3$. We will find that the current catalog of SLSN 
Ic's suitable for this study already confirms the tendencies discussed above, though the statistics are not yet good enough to strongly differentiate between these two competing models. We show in $\S 4$ how large the source catalog needs to be in order to rule out one or the other expansion scenario at a 3-sigma confidence level, and we present our conclusions in $\S 5$.

\section{Methodology}

Eleven of the SLSN Ic's identified by Inserra \& Smartt (2014) are appropriate for this work, and we base our analysis on the methodology described in their paper and in Li et al. (2014). Briefly, the chosen SLSN Ic's must have well sampled light-curves around peak luminosity, and photometric coverage from several days pre-maximum to 30 days (in the rest frame) after the peak. This time delay appears to be optimal for use in the Phillips relation (Inserra \& Smartt 2014).

All 11 of these events appear to be similar to the well-observed SN2010gx, and these decay rapidly after peak brightness. They belong to the group of 2005ap-like events, the first such SN discovered in this category. Other SLSN Ic's could not be included simply because of lack of sufficient temporal coverage, even though their identification has been securely classified in previous work (see, e.g., Leloudas et al. 2012; Quimby et al 2011; Inserra et al. 2013; Chomiuk et al. 2011; Berger et al. 2012; Nicholl et al. 2014).

The SNe listed in Table 1 were located in faint, dwarf galaxies, and were unlikely to have suffered significant extinction beyond the reddening induced by interstellar dust in our Galaxy. Thus, in all cases, a standard reddening curve was assumed with $R_{V}=A_{V} / E(B-V)=3.1$. In addition, the redshift coverage is quite large $(0.143<z<1.206)$, so $K$-correction and time dilation effects were included in order to 


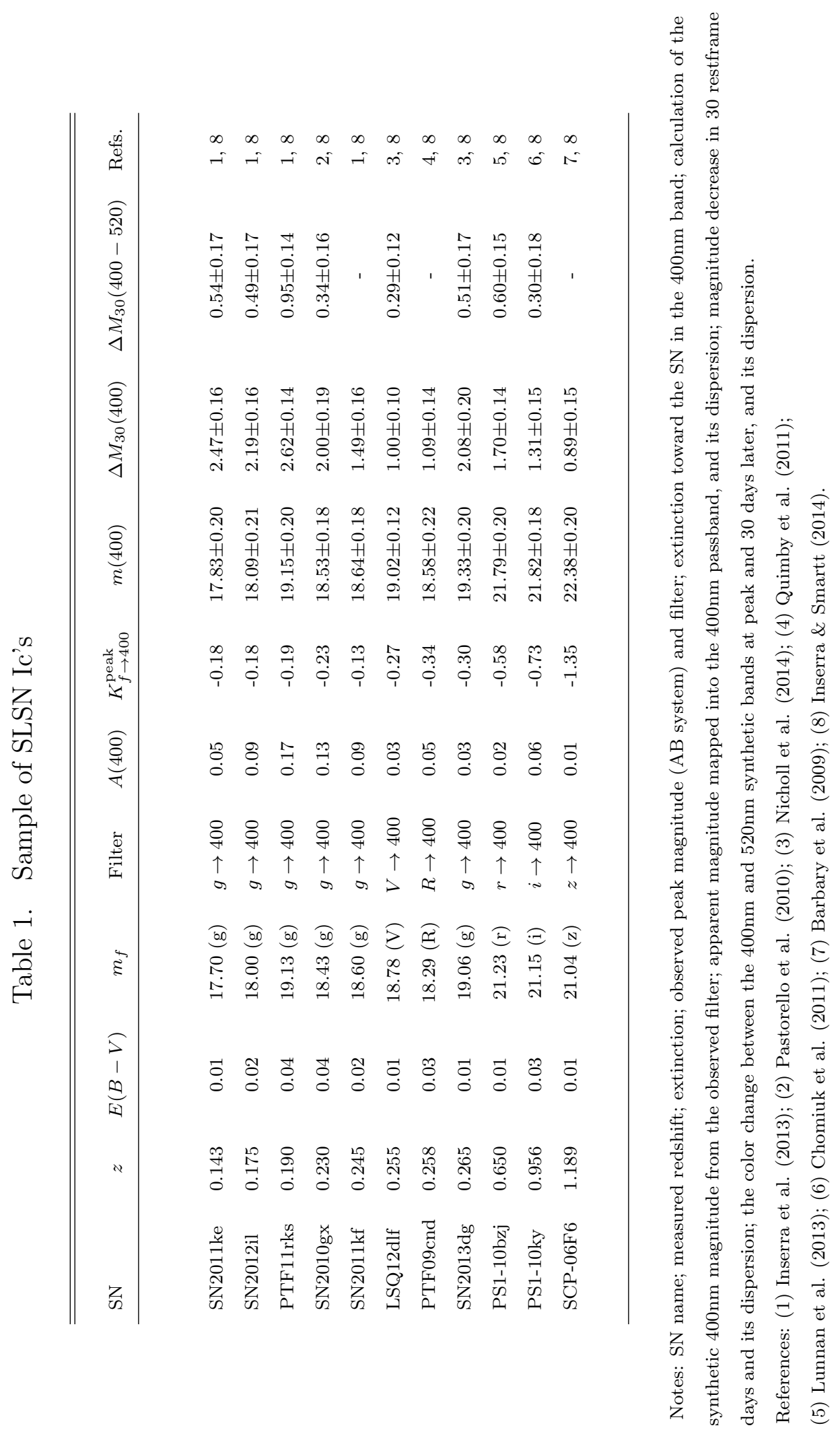


obtain the absolute rest-frame peak magnitudes. These are listed in the table, along with the source names, their redshifts, apparent peak magnitudes (and filters), the magnitude decrease over 30 days, and the color change between the 400nm and 520nm synthetic (restframe) bands at peak and 30 days later.

In order to provide consistent, standarized comparative rest frame properties, the observed apparent magnitudes ( $m$ in Table 1 ) have been converted into defined, synthetic magnitudes. Since the SLSN Ic spectrum around 400nm is continuum dominated, Inserra \& Smartt (2014) defined a synthetic passband with an effective width of 80nm, centered at wavelength $400 \mathrm{~nm}$, having steep wings and a flat top. In Table 1, this is referred to as the 400nm band. All of the chosen events in this table have sufficient photometric coverage to allow the $K$-correction to uniformly map the observed filter's wavelength range to this 400nm bandpass in the rest frame. The absolute magnitudes are then formally defined by the relation

$$
\begin{aligned}
M_{\text {peak }}(400) & =m(400)-\mu \\
& =m_{f}-K_{f \rightarrow 400}^{\text {peak }}-A(400)-\mu,
\end{aligned}
$$

where $m(400)$ is the apparent magnitude mapped into the restframe $400 \mathrm{~nm}$ band, $\mu$ is the distance modulus calculated from the luminosity distance, $m_{f}$ is the AB magnitude in the observed filter $f(g, V, R, r, z$, or $i$, as indicated in Table 1), A(400) is the Galactic extinction, and $K_{f \rightarrow 400}^{\text {peak }}$ is the $K$-correction from the observed filter in Table 1 to the synthetic $400 \mathrm{~nm}$ bandpass. The values of $A(400)$ and $m(400)$ are listed in Table 1 . Note that $m(400)$ is a cosmology-independent apparent magnitude.

The peak magnitude-decline relation for SLSN Ic in the rest frame 400nm band is (Inserra \& Smartt 2014)

$$
M_{\text {peak }}(400)=M_{0}+\alpha \Delta M_{30}(400)
$$


where $\alpha$ is the slope, $\Delta M_{30}(400)$ is the decline at 30 days, and $M_{0}$ is a constant representing the absolute peak magnitude at $\Delta M_{30}(400)=0$. Inserra \& Smartt (2014) also found that $M_{\text {peak }}(400)$ appears to have a strong color dependence. Redder objects are fainter and also become redder faster. This peak magnitude-color evolution relation is given by

$$
M_{\text {peak }}(400)=M_{0}+\alpha \Delta M_{30}(400-520)
$$

where $\Delta M_{30}(400-520)$ is the difference in color $M(400)-M(520)$ between the peak and 30 days later. (Note that $M_{0}$ and $\alpha$ need not be the same in these two expressions.)

With Equations (2) and (3), an effective (standardized) apparent magnitude $m^{\text {eff }}$ may be obtained as follows:

$$
m^{\mathrm{eff}} \equiv m(400)-\alpha \Delta M_{30}
$$

The term $\alpha \Delta M_{30}$ represents an adjustment due to the peak magnitude-decline relation, in terms of $\alpha \Delta M_{30}(400)$, or the peak magnitude-color evolution relation, in terms of $\alpha \Delta M_{30}(400-520)$, as the case may be. The effective apparent magnitude may also be expressed as (Perlmutter et al. 1997,1999)

$$
m^{\mathrm{eff}}=\Upsilon+5 \log _{10}\left[H_{0} d_{L}(z)\right]
$$

where $H_{0}$ is the Hubble constant in units of $\mathrm{km} \mathrm{s}^{-1} \mathrm{Mpc}^{-1}$ and $d_{L}$ is the luminosity distance in units of Mpc. Here $\Upsilon$ is the " $H_{0}$-free" $400 \mathrm{~nm}$ absolute peak magnitude, represented in terms of the standardizable absolute magnitude $M_{0}$, according to the definition

$$
\Upsilon \equiv M_{0}-5 \log _{10}\left(H_{0}\right)+25
$$

(see Li et al. 2014 and references cited therein).

The apparent correlations suggest the use of two "nuisance" parameters ( $\alpha$ and $\Upsilon$ ) whose optimization along with the model parameters decreases the overall scatter in the 
distance modulus. For each model, we therefore find the best fit by minimizing the $\chi^{2}$ statistic, defined as follows:

$$
\chi^{2}=\sum_{i} \frac{\left[m_{i}(400)-\alpha \Delta M_{30, i}-\Upsilon-5 \log _{10}\left[H_{0} d_{L}\left(z_{i}\right)\right]\right]^{2}}{\sigma_{m_{i}(400)}^{2}+\alpha^{2} \sigma_{\Delta M_{30, i}}^{2}} .
$$

In $\Lambda$ CDM, the luminosity distance $d_{L}^{\Lambda \mathrm{CDM}}$ depends on several parameters, including $H_{0}$ and the mass fractions $\Omega_{\mathrm{m}} \equiv \rho_{\mathrm{m}} / \rho_{\mathrm{c}}, \Omega_{\mathrm{r}} \equiv \rho_{\mathrm{r}} / \rho_{\mathrm{c}}$, and $\Omega_{\mathrm{de}} \equiv \rho_{\mathrm{de}} / \rho_{\mathrm{c}}$, defined in terms of the current matter $\left(\rho_{\mathrm{m}}\right)$, radiation $\left(\rho_{\mathrm{r}}\right)$, and dark-energy $\left(\rho_{\mathrm{de}}\right)$ densities, and the critical density $\rho_{\mathrm{c}} \equiv 3 c^{2} H_{0}^{2} / 8 \pi G$. Assuming zero spatial curvature, so that $\Omega_{\mathrm{m}}+\Omega_{\mathrm{r}}+\Omega_{\mathrm{de}}=1$, the luminosity distance to redshift $z$ is given by the expression

$$
d_{L}^{\Lambda \mathrm{CDM}}(z)=\frac{c}{H_{0}}(1+z) \int_{0}^{z}\left[\Omega_{\mathrm{m}}(1+z)^{3}+\Omega_{\mathrm{r}}(1+z)^{4}+\Omega_{\mathrm{de}}(1+z)^{3\left(1+w_{\mathrm{de}}\right)}\right]^{-1 / 2} d z
$$

where $p_{\text {de }}=w_{\text {de }} \rho_{\text {de }}$ is the dark-energy equation of state. The Hubble constant $H_{0}$ cancels out in Equation (7) when we multiply $d_{L}$ by $H_{0}$, so the essential remaining parameters in flat $\Lambda \mathrm{CDM}$ are $\Omega_{\mathrm{m}}$ and $w_{\mathrm{de}}$. If we further assume that dark energy is a cosmological constant with $w_{\mathrm{de}}=-1$, then only the parameter $\Omega_{\mathrm{m}}$ is available to fit the data.

In the $R_{\mathrm{h}}=$ ct Universe (Melia 2007; Melia \& Abdelqader 2009; Melia \& Shevchuk 2012), the luminosity distance depends only on $H_{0}$, but since here too the Hubble constant cancels out in the product $H_{0} d_{L}$, there are actually no free (model) parameters left to fit the SLSN Ic data. In this cosmology,

$$
d_{L}^{R_{\mathrm{h}}=c t}(z)=\frac{c}{H_{0}}(1+z) \ln (1+z)
$$

\section{Results}

We have assumed that SLSN Ic's can be used as standardizable candles and applied the $\Delta M_{30}$ decline relation (with 11 objects) and the peak magnitude-color evolution relation 
(with 8 objects) to compare the standard $(\Lambda \mathrm{CDM})$ model with the $R_{\mathrm{h}}=c t$ Universe. In this section, we discuss how the fits have been optimized, first for $\Lambda \mathrm{CDM}$, and then for $R_{\mathrm{h}}=c t$. The outcome for each model is more fully described and discussed in subsequent sections.

\section{1. $\Lambda \mathrm{CDM}$}

In the most basic $\Lambda \mathrm{CDM}$ model, the dark-energy equation of state parameter, $w_{\mathrm{de}}$, is exactly -1 . The Hubble constant $H_{0}$ cancels out in Equation $(7)$ when we multiply $d_{L}$ by $H_{0}$, so the essential remaining parameter is $\Omega_{\mathrm{m}}$. Type Ia SN measurements (see, e.g., Perlmutter et al. 1998, 1999; Riess et al. 1998; Schmidt et al. 1998), CMB anisotropy data (e.g., Hinshaw et al. 2013), and baryon acoustic oscillation (BAO) peak length scale estimates (e.g., Samushia \& Ratra 2009), strongly suggest that we live in a spatially flat, dark energy-dominated universe with concordance parameter values $\Omega_{\mathrm{m}} \approx 0.27$ and $H_{0} \approx 70$ $\mathrm{km} \mathrm{s}^{-1} \mathrm{Mpc}^{-1}$.
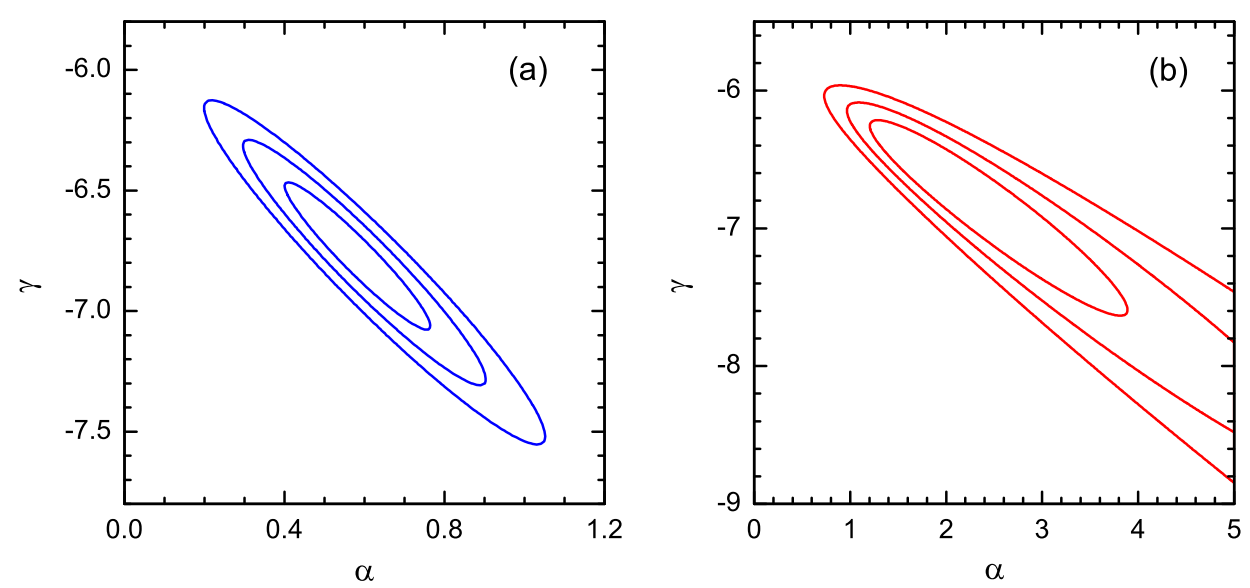

Fig. 1. - 1-3 $\sigma$ constraints on $\alpha$ and $\Upsilon$ for the concordance model, using the $\Delta M_{30}$ decline relation (panel a) and the peak magnitude-color evolution relation (panel b). 
We will therefore first attempt to fit the data with this concordance model, using prior values for all the model parameters, but not the two "nuisance" parameters $\alpha$ and $\Upsilon$. For the $\Delta M_{30}$ decline relation (with 11 objects), the resulting constraints on $\alpha$ and $\Upsilon$ are shown in Figure 1(a). For this fit, we obtain $\alpha=0.57_{-0.17}^{+0.19}(1 \sigma), \Upsilon=-6.75_{-0.32}^{+0.28}(1 \sigma)$, and a $\chi^{2}$ per degree of freedom of $\chi_{\text {dof }}^{2}=27.04 / 9=3.00$, remembering that all of the $\Lambda$ CDM parameters are assumed to have prior values, except for the "nuisance" parameters $\alpha$ and $\Upsilon$. (The corresponding data and best fit are shown in the top lefthand panel of Figure 4.) For the peak magnitude-color evolution relation (with 8 objects), the resulting constraints on $\alpha$ and $\Upsilon$ are shown in Figure 1(b). The best-fit parameters are $\alpha=2.00_{-0.79}^{+1.91}(1 \sigma)$, $\Upsilon=-6.65_{-0.97}^{+0.45}(1 \sigma)$. The $\chi^{2}$ per degree of freedom for the concordance model with these optimized "nuisance" parameters is $\chi_{\text {dof }}^{2}=2.48 / 6=0.41$ (see also the top righthand panel in Figure 4).

If we relax some of the priors, and allow $\Omega_{\mathrm{m}}$ to be a free parameter, we obtain the $1-3 \sigma$ constraint contours shown in the top panel of Figure 2 for the $\Delta M_{30}$ decline relation, and the bottom panel for the peak magnitude-color evolution relation. These contours show that at the $1 \sigma$ level, the optimized parameter values are $\alpha=0.46_{-0.18}^{+0.18}, \Upsilon=-6.41_{-0.33}^{+0.34}$, and $\Omega_{m}=0.62_{-0.34}^{+0.35}$. We find that the $\chi^{2}$ per degree of freedom for the optimized flat $\Lambda$ CDM model is $\chi_{\text {dof }}^{2}=24.89 / 8=3.11$. The bottom panel of Figure 2 shows the $1-3 \sigma$ constraints on $\alpha, \Upsilon$, and $\Omega_{\mathrm{m}}$ for the flat $\Lambda \mathrm{CDM}$ model, using the peak magnitude-color evolution relation. The contours show that at the $1 \sigma$-level, $\alpha=2.05_{-0.94}^{+1.19}, \Upsilon=-6.52_{-0.68}^{+0.50}$, but that $\Omega_{\mathrm{m}}$ is poorly constrained; only a lower limit of $\sim 0.05$ can be set at this confidence level and the best-fit $\Omega_{\mathrm{m}}$ is 0.59 . The $\chi^{2}$ per degree of freedom is $\chi_{\text {dof }}^{2}=2.15 / 5=0.43$. 

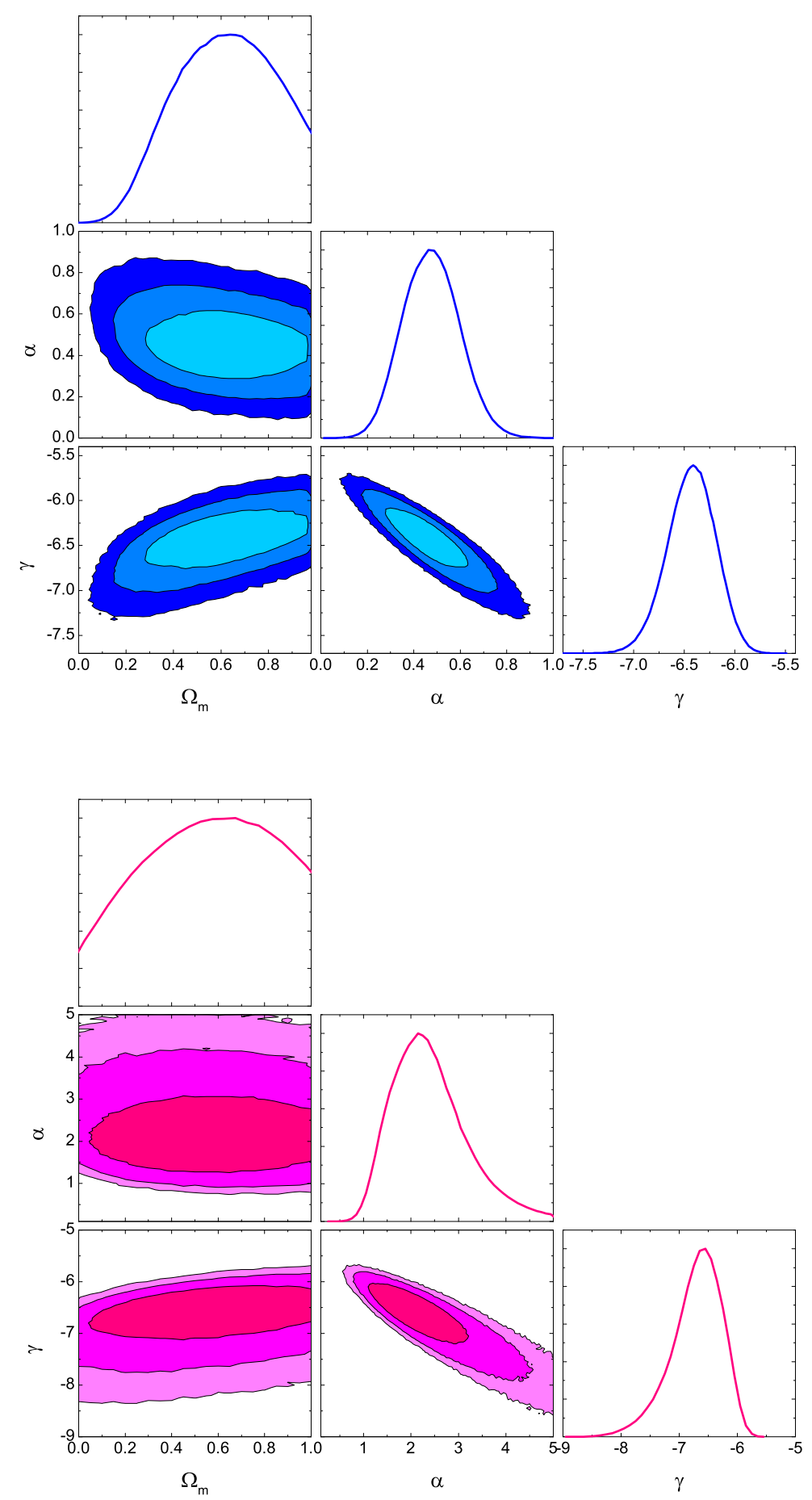

Fig. 2.- Top panel: Constraints on $\alpha, \Upsilon$ and $\Omega_{\mathrm{m}}$ for the flat $\Lambda \mathrm{CDM}$ model, using the $\Delta M_{30}$ decline relation. Bottom panel: Same as the top panel, but using the peak magnitude-color evolution relation. 


\subsection{The $R_{\mathrm{h}}=c t$ Universe}

The $R_{\mathrm{h}}=c t$ Universe has only one free parameter, $H_{0}$, but since the Hubble constant cancels out in the product $H_{0} d_{L}$, there are actually no free (model) parameters left to fit the SLSN Ic data. The results of fitting the $\Delta M_{30}$ decline relation with this cosmology are shown in Figure 3(a). We see here that the best fit corresponds to $\alpha=0.50_{-0.17}^{+0.19}$ $(1 \sigma)$ and $\Upsilon=-6.48_{-0.31}^{+0.28}(1 \sigma)$. With $11-2=9$ degrees of freedom, the reduced $\chi^{2}$ is $\chi_{\text {dof }}^{2}=25.79 / 9=2.87$. The results of fitting the peak magnitude-color evolution relation with this cosmology are shown in Figure 3(b). We see here that the best fit corresponds to $\alpha=1.88_{-0.78}^{+1.84}(1 \sigma)$ and $\Upsilon=-6.44_{-0.94}^{+0.44}(1 \sigma)$. With $8-2=6$ degrees of freedom, the reduced $\chi^{2}$ is $\chi_{\text {dof }}^{2}=2.19 / 6=0.37$.
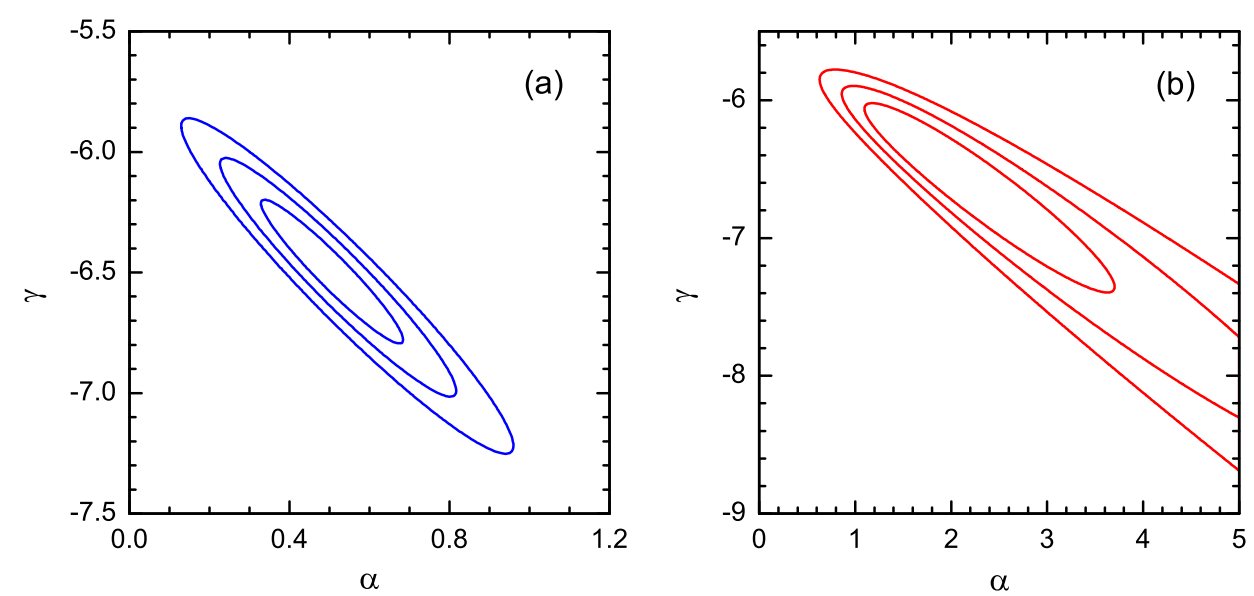

Fig. 3.- 1-3 $\sigma$ constraints on $\alpha$ and $\Upsilon$ for the $R_{\mathrm{h}}=c t$ Universe, using the $\Delta M_{30}$ decline relation (panel a) and the peak magnitude-color evolution relation (panel b).

To facilitate a direct comparison between $\Lambda \mathrm{CDM}$ and $R_{\mathrm{h}}=c t$, we show in Figure 4 the Hubble diagrams for SLSN Ic's. In the left panel of Figure 4, the effective magnitudes $m^{\text {eff }}$ of 11 SLSN Ic are plotted as solid points, together with the best-fit theoretical curves (from top to bottom) for the concordance model (with prior values of the parameters, and with 

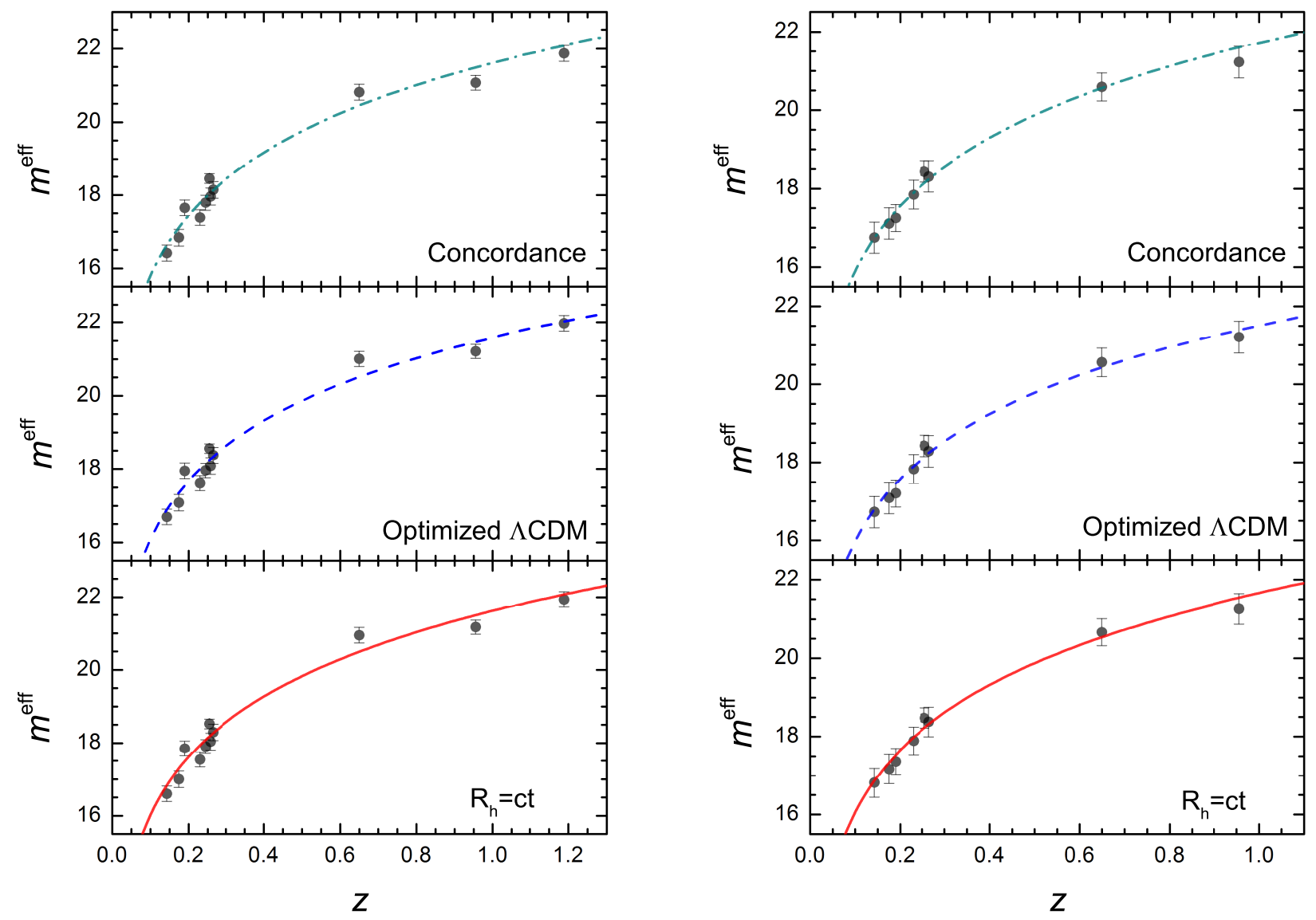

Fig. 4.- Hubble diagrams for SLSN Ic. The effective apparent magnitudes of SLSN Ic are plotted as solid points, together with their corresponding best-fit theoretical curves. Left: The effective magnitudes are calculated using the $\Delta M_{30}$ decline relation. Right: The effective magnitudes are calculated using the peak magnitude-color evolution relation. 
$\alpha=0.57$ and $\Upsilon=-6.75$ ), for the optimized flat $\Lambda$ CDM model (with $\Omega_{\mathrm{m}}=0.62$, and with $\alpha=0.46$, and $\Upsilon=-6.41$ ). and for the $R_{\mathrm{h}}=c t$ Universe (with $\alpha=0.50$ and $\Upsilon=-6.48$ ). The Hubble diagrams derived using the peak magnitude-color evolution relation are shown in the righthand panels of Figure 4 . Here, the effective magnitudes $m^{\text {eff }}$ of 8 SLSN Ic are plotted as solid points, together with the best-fit theoretical curves for the concordance model (with prior values of the parameters, and with $\alpha=2.00$ and $\Upsilon=-6.65$ ), for the optimized flat $\Lambda$ CDM model (with $\Omega_{\mathrm{m}}=0.59$, and with $\alpha=2.05$, and $\Upsilon=-6.52$ ), and for the $R_{\mathrm{h}}=c t$ Universe (with $\alpha=1.88$ and $\Upsilon=-6.44$ ). Strictly based on their $\chi_{\text {dof }}^{2}$ values, the optimized $\Lambda$ CDM model and the $R_{\mathrm{h}}=c t$ Universe appear to fit the SLSN Ic data comparably well. However, because these models formulate their observables (such as the luminosity distances in Equations 8 and 9) differently, and because they do not have the same number of free parameters, a comparison of the likelihoods for either being closer to the 'true' model must be based on model selection tools.

\subsection{Model Selection Tools}

Several information criteria commonly used in cosmology (see, e.g., Melia \& Maier 2013, and references cited therein) include the Akaike Information Criterion, $\mathrm{AIC}=\chi^{2}+2 n$, where $n$ is the number of free parameters (Akaike 1973; Takeuchi 2000; Liddle 2004, 2007; Tan \& Biswas 2012), the Kullback Information Criterion, KIC $=\chi^{2}+3 n$ (Bhansali \& Downnham 1977; Cavanaugh 1999, 2004), and the Bayes Information Criterion, BIC $=\chi^{2}+(\ln N) n$, where $N$ is the number of data points (Schwarz 1978; Liddle et al. 2006; Liddle 2007). With $\mathrm{AIC}_{\alpha}$ characterizing model $\mathcal{M}_{\alpha}$, the unnormalized confidence that this model is true is the Akaike weight $\exp \left(-\mathrm{AIC}_{\alpha} / 2\right)$. Model $\mathcal{M}_{\alpha}$ has likelihood

$$
P\left(\mathcal{M}_{\alpha}\right)=\frac{\exp \left(-\mathrm{AIC}_{\alpha} / 2\right)}{\exp \left(-\mathrm{AIC}_{1} / 2\right)+\exp \left(-\mathrm{AIC}_{2} / 2\right)}
$$


of being the correct choice in this one-on-one comparison. The difference $\Delta \mathrm{AIC} \equiv$ $\mathrm{AIC}_{2}-\mathrm{AIC}_{1}$ determines the extent to which $\mathcal{M}_{1}$ is favoured over $\mathcal{M}_{2}$. For Kullback and Bayes, the likelihoods are defined analogously.

With the optimized fits we have reported in this paper, our analysis of the $\Delta M_{30}$ decline relation (with 11 objects) shows that $R_{\mathrm{h}}=c t$ is favoured over the flat $\Lambda$ CDM model with a likelihood of $\approx 63.4 \%$ versus $36.6 \%$ using $\mathrm{AIC}, \approx 74.1 \%$ versus $\approx 25.9 \%$ using $\mathrm{KIC}$, and $\approx 67.9 \%$ versus $\approx 32.1 \%$ using BIC. In our one-on-one comparison using the peak magnitude-color evolution relation (with 8 objects), the $R_{\mathrm{h}}=c t$ Universe is preferred over $\Lambda \mathrm{CDM}$ with a likelihood of $\approx 72.7 \%$ versus $27.3 \%$ using $\mathrm{AIC}, \approx 81.5 \%$ versus $\approx 18.5 \%$ using $\mathrm{KIC}$, and $\approx 73.5 \%$ versus $\approx 26.5 \%$ using $\mathrm{BIC}$.

\section{Monte Carlo Simulations with a Mock Sample}

These results are interesting, though the current sample of SLSN Ic's is clearly too small for either model to be ruled out just yet. However, this situation will change with the discovery of new SNe, particularly at redshifts $z>2$. To anticipate how well the SLSN Ic catalog obeying the peak magnitude-color evolution relation may be used to constrain the dark-energy equation of state in $\Lambda \mathrm{CDM}$, and to differentiate between this standard model and the $R_{\mathrm{h}}=c t$ Universe, we will here produce mock samples of SLSN Ic's based on the current measurement accuracy. In using the model selection tools, the outcome $\Delta \equiv \mathrm{AIC}_{1}-$ $\mathrm{AIC}_{2}$ (and analogously for $\mathrm{KIC}$ and $\mathrm{BIC}$ ) is judged 'positive' in the range $\Delta=2-6$, 'strong' for $\Delta=6-10$, and 'very strong' for $\Delta>10$. In this section, we will estimate the sample size required to significantly strengthen the evidence in favour of $R_{\mathrm{h}}=c t$ or $\Lambda \mathrm{CDM}$, by conservatively seeking an outcome even beyond $\Delta \simeq 11.62$, i.e., we will see what is required to produce a likelihood $\sim 99.7 \%$ versus $\sim 0.3 \%$, corresponding to a $3 \sigma$ confidence level. 
We will consider two cases: one in which the background cosmology is assumed to be $\Lambda \mathrm{CDM}$, and a second in which it is $R_{\mathrm{h}}=c t$, and we will attempt to estimate the number of SLSN Ic's required in each case to rule out the alternative (presumably incorrect) model at a $\sim 99.7 \%$ confidence level. The synthetic SLSN Ic's are each characterized by a set of parameters denoted as $\left(z, m[400], \Delta M_{30}[400-520]\right)$. We generate the synthetic sample using the following procedure:

1. Since a subclass of broad-lined Type Ic SNe are observed to be associated with Gamma-ray bursts (GRBs; Hjorth et al. 2003; Stanek et al. 2003), we simulate the $z$-distribution of our sample based on the observed $z$-distribution of GRBs. Shao et al. (2011) found that the $z$-distribution of GRBs appears to be asymptotic to a parameter-free probability density distribution, $f(z)=z e^{-z}$. The redshift $z$ of our SLSN Ic events is generated randomly from this function. Since they can be discovered out to redshifts $z \sim 4$ (e.g., Chomiuk et al. 2011; Cooke et al. 2012; Berger et al. 2012), the range of redshifts for our analysis is $[0,4]$. We assign the absolute peak magnitude $M(400)$ uniformly between -23.0 and $-21.0 \mathrm{mag}$, based on the current SLSN Ic measurements.

2. The synthetic apparent peak magnitude $m(400)$ is calculated using the relation $m(400)=M(400)+\mu(z)$, where $\mu(z)$ is the distance modulus at $z$, for a flat $\Lambda$ CDM cosmology with $\Omega_{\mathrm{m}}=0.27$ and $H_{0}=70 \mathrm{~km} \mathrm{~s}^{-1} \mathrm{Mpc}^{-1}$ (§ 4.1), or the $R_{\mathrm{h}}=c t$ Universe with $H_{0}=70 \mathrm{~km} \mathrm{~s}^{-1} \mathrm{Mpc}^{-1}(\S 4.2)$.

3. With the selected $z$ and $M(400)$ values, we first infer a $\Delta M_{30}(400-520)$ from the peak magnitude-color evolution relation in a flat $\Lambda \mathrm{CDM}$ cosmology with $\Omega_{\mathrm{m}}=0.27$ and $H_{0}=70 \mathrm{~km} \mathrm{~s}^{-1} \mathrm{Mpc}^{-1}(\S 4.1)$, or the $R_{\mathrm{h}}=$ ct Universe with $H_{0}=70 \mathrm{~km} \mathrm{~s}^{-1} \mathrm{Mpc}^{-1}(\S 4.2$ ). And then we add scatter to this relation by assigning a dispersion to the $\Delta M_{30}(400-520)$ value; i.e., we randomly map this quantity to the new value $\Delta M_{30}^{\prime}(400-520)$ assuming a normal distribution with a center at $\Delta M_{30}(400-520)$ and a dispersion $\sigma=0.1$ mag. This 
value of $\sigma$ is typical for the current (observed) peak magnitude-color evolution relation, which yields a standard deviation $\sigma=0.08$ mag for the linear fit (Inserra \& Smartt 2014). If $\Delta M_{30}^{\prime}(400-520)>0.0 \mathrm{mag}$, this SLSN Ic is included in our sample. Otherwise, it is excluded.

4. We next assign "observational" errors to $m(400)$ and $\Delta M_{30}^{\prime}(400-520)$. We will assign a dispersion $\sigma_{i}=0.05+0.05 k_{i}$ to each event $i$, where $k_{i}$ is a random number between 0 and 1.

This sequence of steps is repeated for each new SLSN Ic in the sample, until we reach the likelihood criterion discussed above. As with the real 11-SLSN Ic sample, we optimize the model fits by minimizing the $\chi^{2}$ function in Equation (7).

\subsection{Assuming $\Lambda \mathrm{CDM}$ as the Background Cosmology}

We have found that a sample of at least 240 SLSN Ic's is required in order to rule out $R_{\mathrm{h}}=c t$ at the $\sim 99.7 \%$ confidence level. The optimized parameters corresponding to the best-fit $w \mathrm{CDM}$ model for these simulated data are displayed in Figure 5. To allow for the greatest flexibility in this fit, we relax the assumption that dark energy is a cosmological constant with $w_{\mathrm{de}}=-1$, and allow $w_{\mathrm{de}}$ to be a free parameter, along with $\Omega_{\mathrm{m}}$. Figure 5 shows the 1-D probability distribution for each parameter $\left(\Omega_{\mathrm{m}}, w_{\mathrm{de}}, \alpha, \Upsilon\right)$, and 2-D plots of the 1-3 $\sigma$ confidence regions for two-parameter combinations. The best-fit values for $w \mathrm{CDM}$ using the simulated sample with $240 \mathrm{SNe}$ in the $\Lambda \mathrm{CDM}$ model are $\Omega_{\mathrm{m}}=0.28_{-0.04}^{+0.03}(1 \sigma)$, $w_{\mathrm{de}}=-1.14_{-0.35}^{+0.28}(1 \sigma), \alpha=2.21_{-0.10}^{+0.10}(1 \sigma)$, and $\Upsilon=-6.80_{-0.13}^{+0.10}(1 \sigma)$.

To gauge the impact of these constraints more clearly, we show in Figure 6 the confidence regions (shaded, with red contours) for $\Omega_{\mathrm{m}}$ and $w_{\text {de }}$ using these 240 simulated SLSN Ic's (the same as the bottom left-hand panel of Figure 5), and compare these to the 


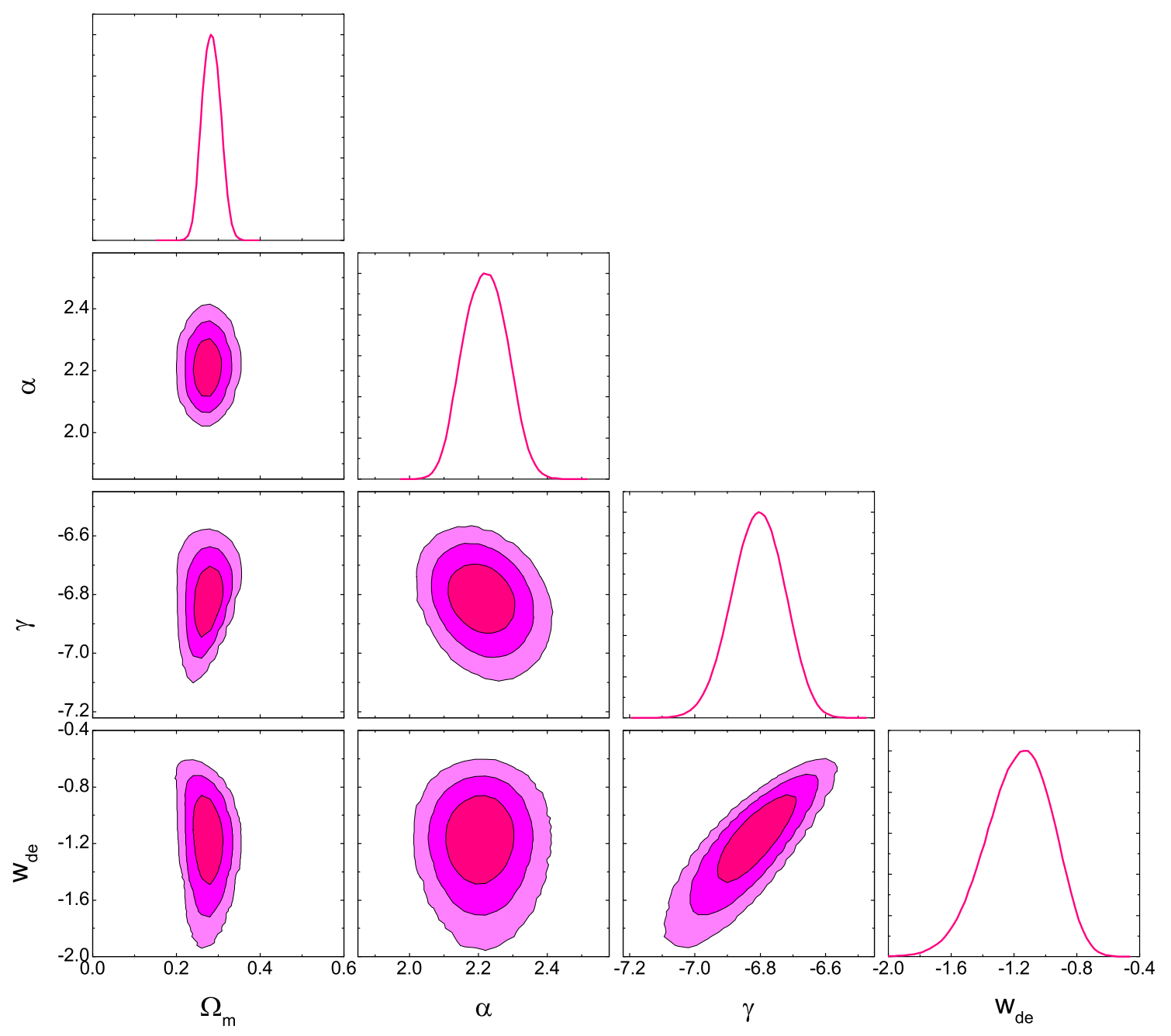

Fig. 5.- The 1-D probability distributions and 2-D regions with the 1-3 $\sigma$ contours corresponding to the parameters $\Omega_{\mathrm{m}}, w_{\mathrm{de}}, \alpha$, and $\Upsilon$ in the best-fit $w$ CDM model, using the simulated sample with 240 SLSN Ic's, assuming $\Lambda$ CDM as the background cosmology. 
constraint contours for the 580 Union2.1 Type Ia SN data (Suzuki et al. 2012) (represented by the blue contours in Figure 6). It is straightforward to see how effectively the SLSN Ic's could be used as a cosmological tool, because the confidence regions resulting from their analysis are smaller (and narrower for $\Omega_{\mathrm{m}}$ ) than those corresponding to the Type Ia events. The better constraints are mainly due to the fact that SLSN Ic's are distributed over a much wider redshift range, extending towards high- $z$, where tighter constraints on the model can be achieved.

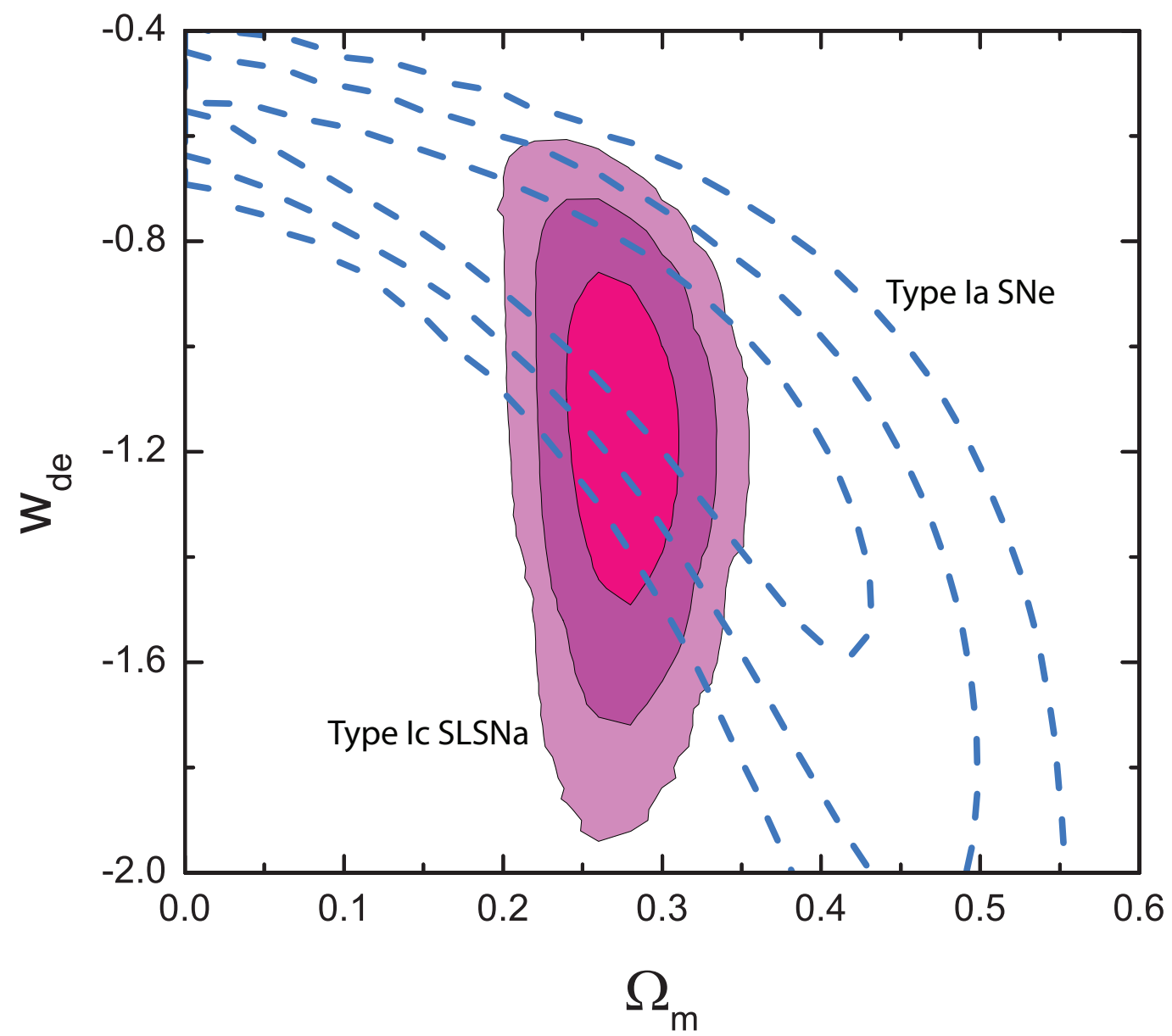

Fig. 6.- One- $\sigma, 2-\sigma$ and 3- $\sigma$ confidence regions for the $w$ CDM model using simulated SLSN Ic's (shaded, with red contours), compared with those (dashed blue contours) associated with the 580 Union2.1 Type Ia SNe. 
In Figure 7, we show the corresponding 2-D contours in the $\alpha-\Upsilon$ plane for the $R_{\mathrm{h}}=c t$ Universe. The best-fit values for the simulated sample are $\alpha=2.22_{-0.08}^{+0.09}(1 \sigma)$ and $\Upsilon=-6.53_{-0.04}^{+0.03}(1 \sigma)$.

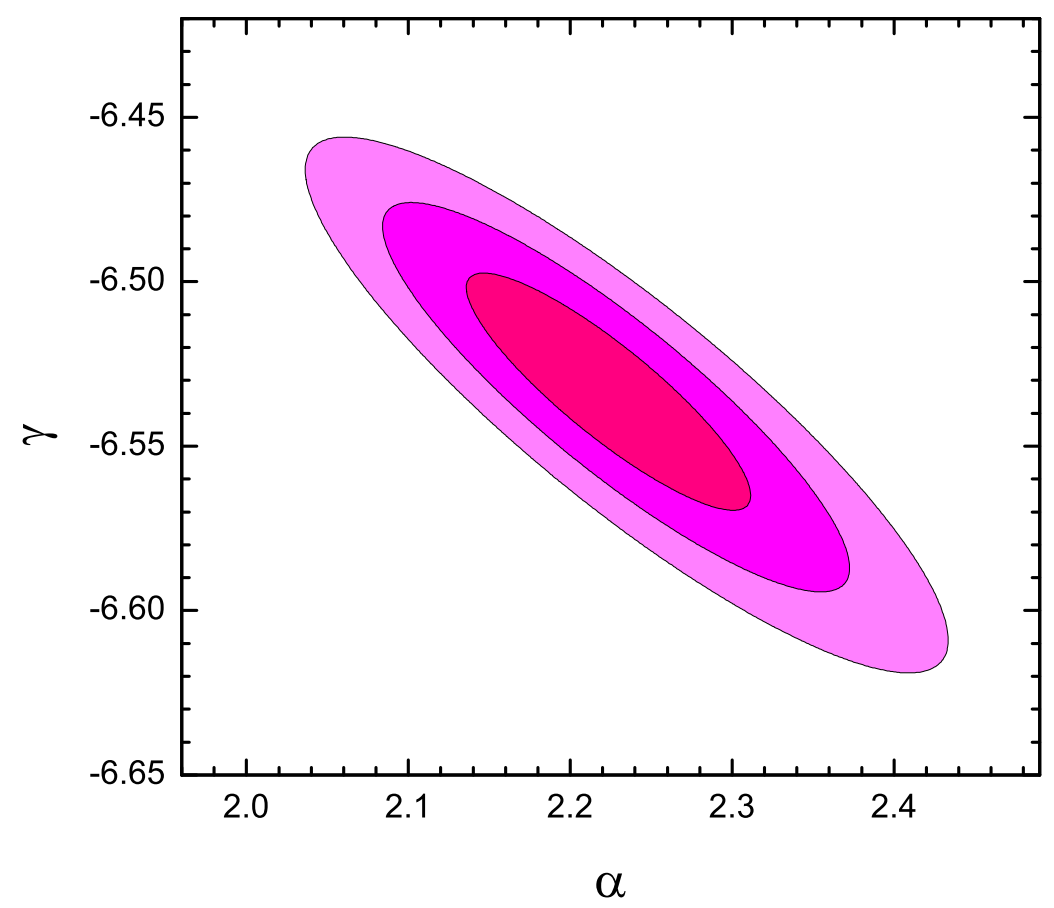

Fig. 7.- The $2-\mathrm{D}$ region with the $1-3 \sigma$ contours for the parameters $\alpha$ and $\Upsilon$ in the $R_{\mathrm{h}}=$ ct Universe, using a sample of 240 SLSN Ic, simulated with $\Lambda$ CDM as the background cosmology. The simulated model parameters were $\Omega_{\mathrm{m}}=0.27, \Omega_{\Lambda}=0.73$, and $H_{0}=70 \mathrm{~km}$ $\mathrm{s}^{-1} \mathrm{Mpc}^{-1}$.

Since the number $N$ of data points in the sample is now much greater than one, the most appropriate information criterion to use is the BIC. The logarithmic penalty in this model selection tool strongly suppresses overfitting if $N$ is large (the situation we have here, which is deep in the asymptotic regime). With $N=240$, our analysis of the simulated sample shows that the BIC would favor the $w \mathrm{CDM}$ model over $R_{\mathrm{h}}=c t$ by an overwhelming 
likelihood of $99.7 \%$ versus only $0.3 \%$ (i.e., the prescribed $3 \sigma$ confidence limit).

\subsection{Assuming $R_{\mathrm{h}}=c t$ as the Background Cosmology}

In this case, we assume that the background cosmology is the $R_{\mathrm{h}}=c t$ Universe, and seek the minimum sample size to rule out $w \mathrm{CDM}$ at the $3 \sigma$ confidence level. We have found that a minimum of 480 SLSN Ic's are required to achieve this goal. To allow for the greatest flexibility in the $w \mathrm{CDM}$ fit, here too we relax the assumption of dark energy as a cosmological constant with $w_{\mathrm{de}}=-1$, and allow $w_{\text {de }}$ to be a free parameter, along with $\Omega_{\mathrm{m}}$. In Figure 8, we show the 1-D probability distribution for each parameter $\left(\Omega_{\mathrm{m}}, w_{\mathrm{de}}\right.$, $\alpha, \Upsilon)$, and $2-\mathrm{D}$ plots of the $1-3 \sigma$ confidence regions for two-parameter combinations. The best-fit values for $w$ CDM using this simulated sample with 480 SLSN Ic's are $\Omega_{\mathrm{m}}=0.0$, $w_{\mathrm{de}}=-0.33_{-0.06}^{+0.01}(1 \sigma), \alpha=2.05_{-0.07}^{+0.07}(1 \sigma)$, and $\Upsilon=-6.52_{-0.05}^{+0.04}(1 \sigma)$. Note that the simulated SLSN Ic's give a good constraint on $w_{\text {de }}$, but a weak constraint on $\Omega_{\mathrm{m}}$; only an upper limit of 0.09 can be set at the $1 \sigma$ confidence level.

The corresponding 2-D contours in the $\alpha-\Upsilon$ plane for the $R_{\mathrm{h}}=c t$ Universe are

shown in Figure 9. The best-fit values for the simulated sample are $\alpha=2.05_{-0.06}^{+0.07}(1 \sigma)$ and $\Upsilon=-6.52_{-0.02}^{+0.02}(1 \sigma)$. These are similar to those in the standard model, but not exactly the same, reaffirming the importance of reducing the data separately for each model being tested. With $N=480$, our analysis of the simulated sample shows that in this case the BIC would favor $R_{\mathrm{h}}=$ ct over $w \mathrm{CDM}$ by an overwhelming likelihood of $99.7 \%$ versus only $0.3 \%$ (i.e., the prescribed $3 \sigma$ confidence limit). 


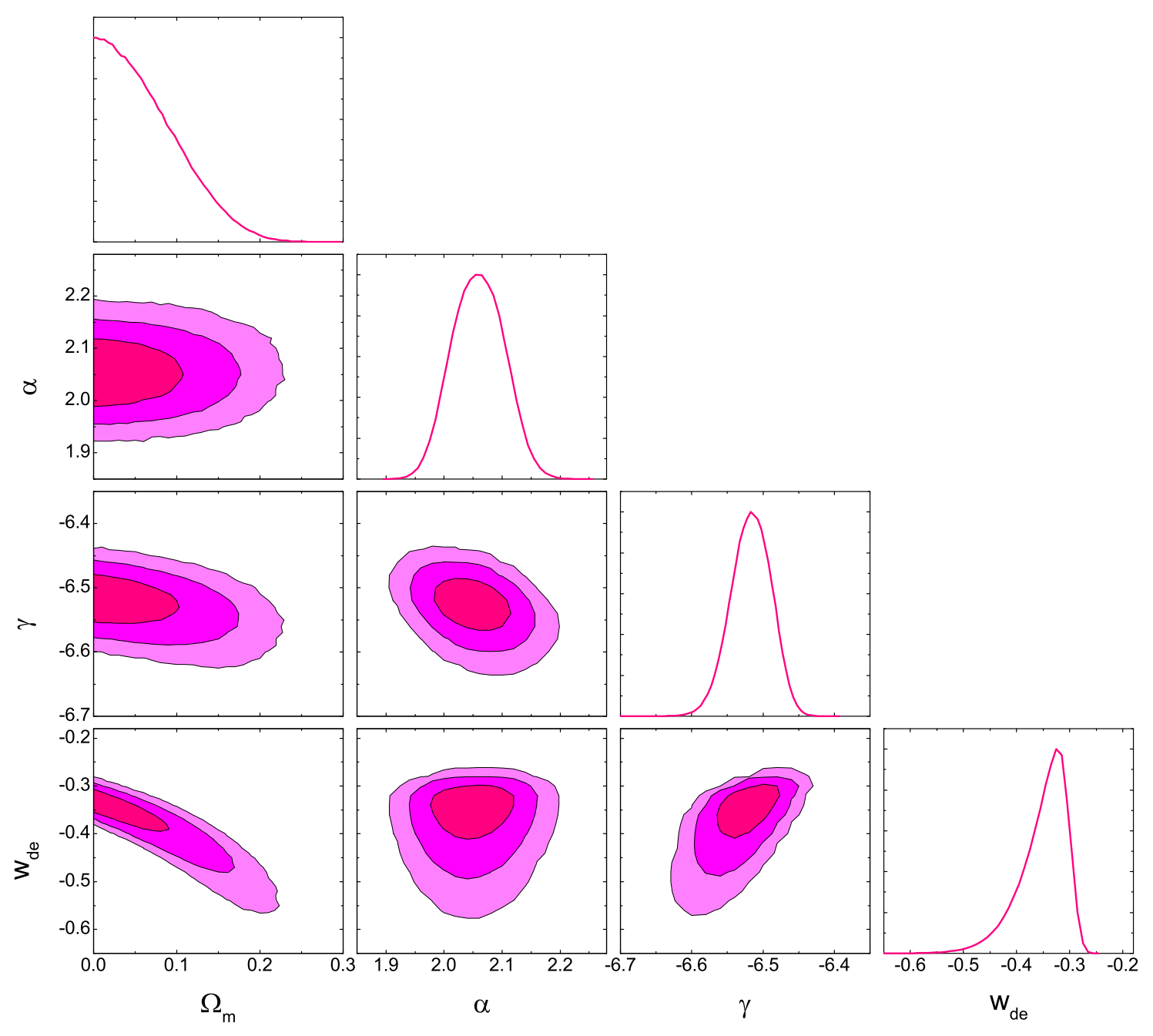

Fig. 8.- Same as Figure 5, except now with $R_{\mathrm{h}}=c t$ as the (assumed) background cosmology. The simulated model parameter was $H_{0}=70 \mathrm{~km} \mathrm{~s}^{-1} \mathrm{Mpc}^{-1}$. 


\section{Conclusions}

It is quite evident that SLSN Ic's may be useful cosmological probes, perhaps even out to redshifts much greater $(z>>2)$ than those accessible using Type Ia SNe. The currently available sample, however, is still quite small; adequate data to extract correlations between empirical, observable quantities, such as lightcurve shape, color evolution and peak luminosity, are available only for tens of events. In this paper, we have proposed to use SLSN Ic's for an actual one-on-one comparison between competing cosmological models. This must be done because the results we have presented here already indicate a strong likelihood of being able to discriminate between models such as $\Lambda$ CDM and $R_{\mathrm{h}}=c t$. Such comparisons have already been made using, e.g., cosmic chronometers (Melia \& Maier 2013), Gamma-ray bursts (Wei et al. 2013), and Type Ia SNe (Wei et al. 2014).

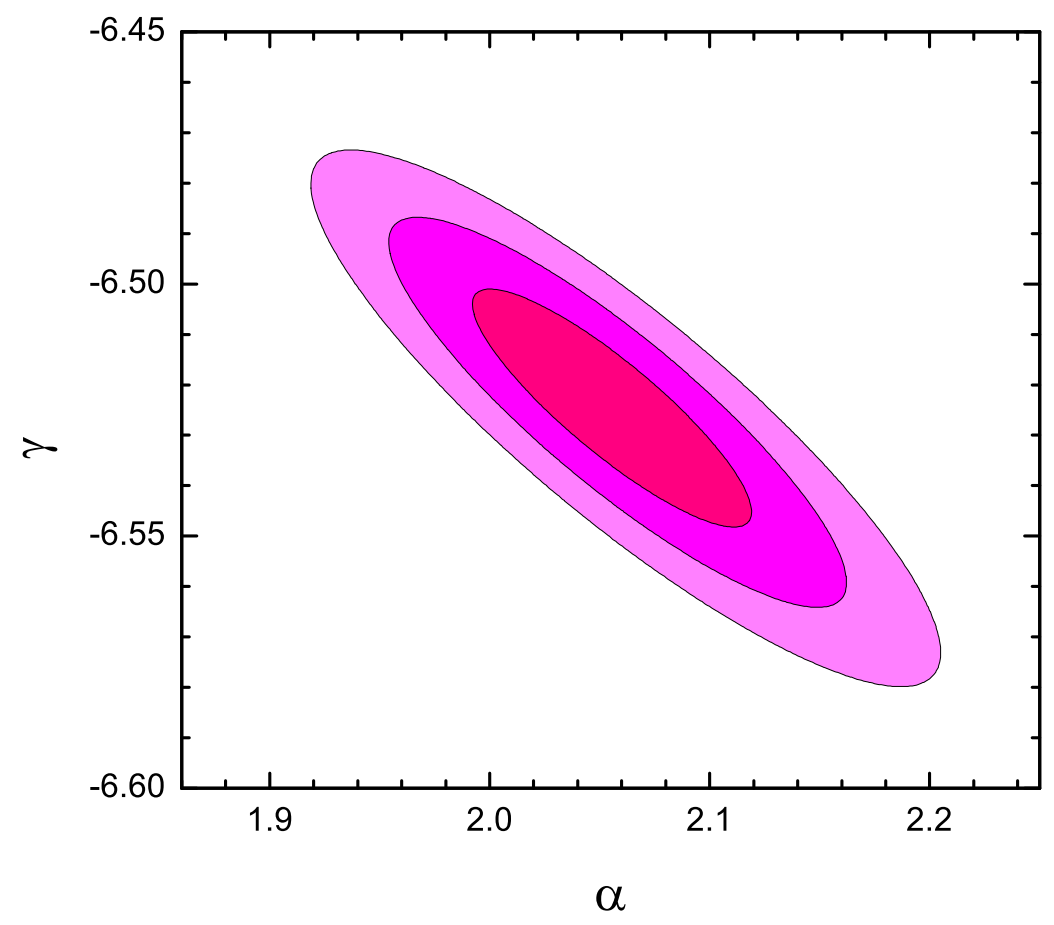

Fig. 9.- Same as Figure 7, except now with $R_{\mathrm{h}}=c t$ as the (assumed) background cosmology. 
We have individually optimized the parameters in each model by minimizing the $\chi^{2}$ statistic. With the optimized fits we have reported above, our analysis of the $\Delta M_{30}$ decline relation (with 11 objects) shows that $R_{\mathrm{h}}=c t$ is favoured over the flat $\Lambda$ CDM model with a likelihood of $\approx 63-74 \%$ versus $26-37 \%$ (depending on the information criterion). In our one-on-one comparison using the peak magnitude-color evolution relation (with 8 objects), the $R_{\mathrm{h}}=c t$ Universe is preferred over $\Lambda \mathrm{CDM}$ with a likelihood of $\approx 73-82 \%$ versus $18-27 \%$.

But though SLSN Ic observations currently tend to favor $R_{\mathrm{h}}=c t$ over $\Lambda$ CDM, the known sample of such measurements is still too small for us to completely rule out either model. We have therefore considered two synthetic samples with characteristics similar to those of the 8 known SLSN Ic measurements, one based on a $\Lambda$ CDM background cosmology, the other on $R_{\mathrm{h}}=c t$. From the analysis of these simulated SLSN Ic's, we have estimated that a sample of about 240 such events are necessary to rule out $R_{\mathrm{h}}=c t$ at a $\sim 99.7 \%$ confidence level if the real cosmology is in fact $\Lambda \mathrm{CDM}$, while a sample of at least $480 \mathrm{SNe}$ would be needed to similarly rule out $w \mathrm{CDM}$ if the background cosmology were instead $R_{\mathrm{h}}=c t$. The difference in required sample size results from $w$ CDM's greater flexibility in fitting the data, since it has a larger number of free parameters.

Our simulations have also shown that a moderate sample size of $\sim 250$ events could reach much tighter constraints on the dark-energy equation of state $w_{\text {de }}$ and on the matter density fraction $\Omega_{\mathrm{m}}$ than are currently available with the 580 Union2.1 Type Ia SNe. If SLSN Ic's can be commonly detected in the future, they have the potential of greatly refining the measurement of cosmological parameters, particularly the dark-energy equation of state $w_{\mathrm{de}}$.

Assembling samples of this size may be feasible with upcoming surveys. For example, the planned survey SUDSS (Survey Using Decam for Super-luminous Supernovae) using 
the Dark Energy Camera on the CTIO Blanco 4m telescope Inserra \& Smartt (2014), and the Subaru/Hyper Suprime-Cam deep survey (Tanaka et al. 2012), have a goal of discovery several hundred SLSNe out to $z \sim 4$ over 3 years by imaging tens of square degrees to a limiting magnitude $\sim 25$ every 2 weeks. These numbers are based on an estimated production in the local universe (Quimby et al. 2013), who reported a rate of $32_{-26}^{+77}$ events $\mathrm{Gpc}^{-3} \mathrm{yr}^{-1}$ at a weighted redshift of $\langle z\rangle=0.17$. This number is low $(0.01 \%)$ compared to that of Type Ia SNe. McCrum et al. (2014) estimate a Type Ic SN rate $\sim 10^{-4}$ of the overall core-collapse SN rate within $0.3<z<1.4$, though this number could be higher at $z>1.5$ (Cooke et al. 2012) due, perhaps, to a decreasing metallicity and increasing cosmic star formation rate. As far as observations from the ground are concerned, assembling a sample of several hundred Type Ic SLSNe over 3 to 5 years therefore looks quite promising, particulary with the surveying capability of LSST (see, e.g., Lien \& Fields 2009), which should cover the whole sky every 2 nights, down to a limiting magnitude $\sim 24$. The expected rate of discovery of core-collapse SNe with this survey is expected to be $\sim 1-2$ $\mathrm{s}^{-1}$ out to a redshift $z \sim 2$. Given that $\sim 10^{-4}$ of these are expected to be Type Ic SNe in this redshift range, one should expect LSST to assemble a $\sim 500$ SLSN sample in less than a year. The situation from space is even more exciting. Detecting Type Ic SLSNe out to redshifts $z \sim 10$ via their restframe $400 \mathrm{~nm}$ and $520 \mathrm{~nm}$ bands is plausible with, e.g., EUCLID (Laureijs et al. 2011), WFIRST and the James Webb Space Telescope (specifically the NIRCam). ${ }^{1}$

A major problem with this approach right now, however, is that one must rely on the use of a luminosity-limited sample of supernovae, i.e. those selected to be super-luminous with peak magnitudes $M_{A B}<-21 \mathrm{mag}$ and integrated burst energies $\sim 10^{51} \mathrm{erg}$ (Inserra \& Smartt 2014), in order to test the luminosity distances. The difficulty is that the luminosity-

\footnotetext{
${ }^{1}$ http://www.stsci.edu/jwst/instruments/nircam/
} 
limited sample may turn out to be different with different background cosmologies, given that their inferred luminosities are themselves dependent on the models. This situation is unlikely to change as the sample grows, so it would be necessary to find a way of identifying these SNe, other than simply through their magnitudes. Fortunately, in the case of $R_{\mathrm{h}}=c t$ versus $\Lambda \mathrm{CDM}$, even though their luminosity distances are formulated differently, it turns out that the ensuing distance measures derived from these are quite similar all the way out to $z \sim 6$. As such, Type Ic SLSNe that make the cut for one model and not the other are the exception rather than the rule. In other words, the luminosity-limit applied to the sample examined here does not bias either model very much. But this may not be true in general, and an alternative method of selection is highly desirable.

We are very grateful to Cosimo Inserra for helpful, clarifying discussions. This work is partially supported by the National Basic Research Program ("973" Program) of China (Grants 2014CB845800 and 2013CB834900), the National Natural Science Foundation of China (grants Nos. 11322328, 11373068, 11173064 and 11233008), the One-Hundred-Talents Program and the Youth Innovation Promotion Association, and the Strategic Priority Research Program "The Emergence of Cosmological Structures" (Grant No. XDB09000000) of the Chinese Academy of Sciences, and the Natural Science Foundation of Jiangsu Province. F.M. is also grateful to Amherst College for its support through a John Woodruff Simpson Lectureship, and to Purple Mountain Observatory in Nanjing, China, for its hospitality while this work was being carried out. This work was partially supported by grant 2012T1J0011 from The Chinese Academy of Sciences Visiting Professorships for Senior International Scientists, and grant GDJ20120491013 from the Chinese State Administration of Foreign Experts Affairs. 


\section{REFERENCES}

Akaike, H. 1973, in Second International Symposium on Information Theory, eds. B. N. Petrov and F. Csáki (Budapest: Akadémiai Kiadó), 267

Baltay, C., Rabinowitz, D., Hadjiyska, E., et al. 2013, PASP, 125, 683

Barbary, K., Dawson, K. S., Tokita, K., et al. 2009, ApJ, 690, 1358

Berger, E., Chornock, R., Lunnan, R., et al. 2012, ApJ, 755, L29

Bhansali, R. J. \& Downham, D. Y. 1977, Biometrika, 64, 547

Cano, Z., \& Jakobsson, P. 2014, arXiv:1409.3570

Cavanaugh, J. E. 1999, Statist. Probab. Lett., 42, 333

Cavanaugh, J. E. 2004, Aust. N. Z. J. Stat., 46, 257

Chomiuk, L., Chornock, R., Soderberg, A. M., et al. 2011, ApJ, 743, 114

Cooke, J., Sullivan, M., Gal-Yam, A., et al. 2012, Nature, 491, 228

Drake, A. J., Djorgovski, S. G., Mahabal, A., et al. 2009, ApJ, 696, 870

Gal-Yam, A. 2012, Science, 337, 927

Hamuy, M., Phillips, M. M., Suntzeff, N. B., et al. 1996, AJ, 112, 2438

Hinshaw, G., Larson, D., Komatsu, E., et al. 2013, ApJS, 208, 19

Hjorth, J., Sollerman, J., Møller, P., et al. 2003, Nature, 423, 847

Inserra, C., Smartt, S. J., Jerkstrand, A., et al. 2013, ApJ, 770, 128

Inserra, C., \& Smartt, S. J. 2014, arXiv:1409.4429 
Kaiser, N., Burgett, W., Chambers, K., et al. 2010, Proc. SPIE, 7733,

Laureijs, R., Amiaux, J., Arduini, S. et al. 2011, arXiv:1110.3193

Leloudas, G., Chatzopoulos, E., Dilday, B., et al. 2012, A\&A, 541, A129

Li, X., Hjorth, J., \& Wojtak, R. 2014, arXiv:1409.3567

Liddle, A. R. 2004, MNRAS, 351, L49

Liddle, A. R. 2007, MNRAS, 377, L74

Liddle, A., Mukherjee, P., \& Parkinson, D. 2006, Astron. \& Geophys., 47, 4.30

Lien, A. \& Fields, B. D. 2009, JCAP, 01, id. 047

Lunnan, R., Chornock, R., Berger, E., et al. 2013, ApJ, 771, 97

McCrum, M., Smartt, S. J., Rest, A. et al. 2014, arXiv:1402.1631

Melia, F. 2007, MNRAS, 382, 1917

Melia, F., \& Abdelqader, M. 2009, International Journal of Modern Physics D, 18, 1889

Melia, F., \& Shevchuk, A. S. H. 2012, MNRAS, 419, 2579

Melia, F. 2013a, A\&A, 553, A76

Melia, F. 2013b, ApJ, 764, 72

Melia, F., \& Maier, R. S. 2013, MNRAS, 432, 2669

Melia, F. 2014, J. Cosmology Astropart. Phys., 1, 27

Nicholl, M., Smartt, S. J., Jerkstrand, A., et al. 2013, Nature, 502, 346

Nicholl, M., Smartt, S. J., Jerkstrand, A., et al. 2014, MNRAS, 444, 2096 
Pastorello, A., Smartt, S. J., Botticella, M. T., et al. 2010, ApJ, 724, L16

Perlmutter, S., Aldering, G., della Valle, M., et al. 1998, Nature, 391, 51

Perlmutter, S., Aldering, G., Goldhaber, G., et al. 1999, ApJ, 517, 565

Perlmutter, S., Gabi, S., Goldhaber, G., et al. 1997, ApJ, 483, 565

Phillips, M. M. 1993, ApJ, 413, L105

Pskovskii, I. P. 1977, Soviet Ast., 21, 675

Quimby, R. M., Castro, F., Gerardy, C. L. et al. 2005, BAAS, 37, \#171.02

Quimby, R. 2006, Central Bureau Electronic Telegrams, 644, 1

Quimby, R. M., Kulkarni, S. R., Kasliwal, M. M., et al. 2011, Nature, 474, 487

Quimby, R. M., Aldering, G., Wheeler, J. C., et al. 2007, ApJ, 668, L99

Quimby, R. M., Werner, M. C., Oguri, M. et al. 2013, ApJ Lett., 768, L20

Rau, A., Kulkarni, S. R., Law, N. M., et al. 2009, PASP, 121, 1334

Riess, A. G., Filippenko, A. V., Challis, P., et al. 1998, AJ, 116, 1009

Rust, B. W. 1974, BAAS, 6, 309

Samushia, L., \& Ratra, B. 2009, ApJ, 703, 1904

Schmidt, B. P., Suntzeff, N. B., Phillips, M. M., et al. 1998, ApJ, 507, 46

Schwarz, G. 1978, Ann. Statist., 6, 461

Shao, L., Dai, Z.-G., Fan, Y.-Z., et al. 2011, ApJ, 738, 19

Smith, N., Li, W., Foley, R. J., et al. 2007, ApJ, 666, 1116 
Stanek, K. Z., Matheson, T., Garnavich, P. M., et al. 2003, ApJ, 591, L17

Suzuki, N., Rubin, D., Lidman, C., et al. 2012, ApJ, 746, 85

Tan, M. Y. J. \& Biswas, R. 2012, MNRAS, 419, 3292

Tanaka, M., Moriya, T. J., Yoshida, N. \& Nomoto, K. 2012, MNRAS, 422, 2675

Takeuchi, T. T. 2000, Ap\&SS, 271, 213

Wei, J.-J., Wu, X.-F., \& Melia, F. 2013, ApJ, 772, 43

Wei, J.-J., Wu, X.-F. \& Melia, F. 2014, AJ, submitted 